\title{
Knowledge translation and UWOMJ's expanded authorship
}

$K T$ [knowledge translation] is of critical importance to health research, as it has become clear that the creation of new knowledge often does not, on its own, lead to widespread implementation or impacts on health.

- Canadian Institutes of Health Research (CIHR) ${ }^{1}$

CIHR, the largest academic health research funding agency in Canada, defines knowledge translation (KT) as "a dynamic and iterative process that includes synthesis, dissemination, exchange and ethically sound application of knowledge to improve the health of Canadians, provide more effective health services and products and strengthen the health care system."' Many more interrelated terms are also frequently tossed around and all carry a similar spirit as that of KT, including knowledge transfer, knowledge exchange, and research implementation. ${ }^{2}$ Well-intentioned scholars have worked very hard at defining the nuanced semantic differences between these buzzwords, and they would likely scold the notion that any of these terms could be lumped together. To complicate matters even more, the use and popularity of these terms varies across geographic regions. In the interest of simplicity, only KT (a more prominent term in Canada) will be discussed here. ${ }^{2}$

While CIHR may be notoriously buried in obscure lingo, on the matter of KT the message to clinicians can be made plain and simple; the dialogue across all elements of the healthcare system needs to be improved. This means better communication between all knowledge users, which in addition to clinicians includes researchers, administrators, and policy makers. Given all that is at stake in healthcare, we should not rely on serendipity alone to guide vital health research towards clinical practise and public health measures.
Currently, KT involves considerable time lags; 17 years seems to be a common estimate for the time that it takes for research evidence to integrate into clinical practice. ${ }^{3}$ If time lags are unavoidable because of the demands of safety and ethics, then it is especially important that KT efforts are initiated by young professionals. Promoting KT early on in a healthcare career will encourage knowledge users to see projects through over the span of their careers. From the perspective of a trainee, it seems clear that greater and earlier emphasis on KT, in the form of curriculum, tools, and resources, would be promising investments in the future of KT.

The UWOMJ team hopes to contribute to KT and towards this aim, we were excited to open up authorship to all healthcare-related graduate students as of this issue. We hope that this expansion might help bring together clinical and research trainees and encourage direct collaboration in the future. It was a great pleasure to see that there was interest in this opportunity and that this new venture proceeded very smoothly. Perhaps it was not by chance that one of the articles in this issue, authored by one of the first research students to publish in the UWOMJ, explicitly stressed the importance of KT. Natalie V. Scime's outstanding article on the use of antidepressant medication during pregnancy concludes that "while continued research on the outcomes and safety of antidepressant use during pregnancy is important, tailored KT efforts should be implemented and prioritized to improve the counselling and decision-making process for everyone involved." ${ }^{\prime 4}$ Without doubt, this applies to all areas of healthcare.

We hope that you enjoy this issue on the theme of drugs, a topic that so often tugs on KT and inter-disciplinary expertise.

Alexander Levit Junior Associate Editor

\section{REFERENCES}

1. Canadian Institutes of Health Research. Guide to knowledge translation planning at CIHR: Integrated and end-of-grant approaches. Ottawa (ON): Canadian Institutes of Health Research; 2012. 34 p. (Catalogue Number R4-11/2012E-PDF)

2. Graham I, Logan J, Harrison M, et al. Lost in knowledge translation: Time for a map? J Contin Educ Health Prof. 2006;26(1):13-24.

3. Morris ZS, Wooding S, Grant J. The answer is 17 years, what is the question: understanding time lags in translational research. J R Soc Med. 2011;104(12):510-20.

4. Scime NV. Antidepressant use during pregnancy: Current attitudes and knowledge translation efforts. UWOMJ. 2016;85(1):12-4. 